\title{
Interaction of bacteria and airway epithelial cells
}

\author{
L. Van Alphen
}

The symposium "Interaction of bacteria and airway epithelial cells" of the 5th European Respiratory Congress in Barcelona, September 1995, was organized in order to update current concepts on the mechanisms of interaction of bacteria with airway mucosa in health and disease. The involvement of a variety of bacterial factors and host receptors, and the host and bacterial responses upon the interaction in health and disease were discussed. The four contributions in the Review Series entitled "Interaction of bacteria and airway epithelial cells", dedicated to the symposium, highlight these important aspects of the host-bacterial interactions.

In their contribution, WILSON et al. [1], published in this issue of the Journal, summarize the host and bacterial components mediating the interaction between airway mucosa and pathogenic bacteria. In health, the lower respiratory tract is not colonized. WILSON et al. show that an efficient mucociliary clearance system is required to defend the bronchial tree against bacterial infection. They also stress the importance of impaired host defence, resulting in damaged epithelium as a target for bacterial interaction. Both the inflammatory process and the presence of bacteria with a "pathogenic personality" contribute to the damaging of the epithelia. Damage includes disruption of ciliary beating, loss of ciliated cells, and exposure of epithelial cell receptors for bacterial adherence. Overproduction of mucus also contributes to bacterial infection, since bacteria bind to and are trapped inside the mucus layer, which is poorly permeable for antibacterial defence factors. The bacteria most frequently encountered are Haemophilus influenzae, Pseudomonas aeruginosa and Streptococcus pneumoniae.

Infecting bacteria express a variety of determinants of virulence, including a large variety of adhesins, cilio- and cytotoxins, exoenzymes degrading immunoglobulins, components stimulating mucus production, cytotoxins and alginate.

DE Bentzmann et al. [2] focus on the interaction of $P$. aeruginosa with respiratory epithelial cells, and the importance of this type of interaction for bacterial persistence in the respiratory tract of cystic fibrosis $(\mathrm{CF})$ patients. A very important finding is the level of the asialylated ganglioside, asialo-GM1 receptor, on the epithelial cells as pilus-mediated adherence of $P$. aeruginosa is increased in epithelial cells of $\mathrm{CF}$ patients compared to healthy individuals. $P$. aeruginosa is, itself, able to increase the level further by production of a neuraminidase, and this effect was only found for epithelial cells of CF patients.

Academic Medical Center, University of Amsterdam, Dept of Medical Microbiology, Meibergdreef 15, 1105 AZ, Amsterdam, The Netherlands.
A similar increase in asialo-gangliosides was observed on respiratory epithelial cells edging a wound, in the process of spreading and migration over the collagen matrix during healing. These are the wound areas colonized by $P$. aeruginosa during healing. These results indicate that the epithelial cell asialo-ganglioside receptor is probably important in the infection, and that the host-bacterial interaction is influenced by the conditions of the host and the bacterial modulation of the system.

The airway mucosa of the patients with chronic bronchitis, and even more so with cystic fibrosis are continuously inflamed. AgACE, [3] discusses the mechanisms and factors of mucosal inflammation, especially the inflammatory response upon bacterial interaction with epithelial cells. Most of the data concern the mucosal inflammatory processes of the urinary tract, since this mucosal site has been studied most extensively. These data are very helpful in understanding inflammatory processes in the lung upon bacterial interaction. The production of proinflammatory mediators by epithelial cells is shown to be dependent on the specificity of the interaction between bacteria and epithelial cells. Pili (type 1 and pap) mediated adherence of Escherichia coli stimulates interleukin-8 (IL-8) production by epithelial cells. AGACE demonstrated nicely in vitro that IL-8 has chemotactic activity for the recruitment of neutrophils to the epithelial cell layer, and that the neutrophils are translocated after upregulation of intracellular adhesion molecule-1 (ICAM-1) during the response through the tight junctions to the apical side of the cell layer. This translocation is dependent on ICAM-1 and Mac-1.

KHAIR et al. [4] describe the current state of knowledge of the effect of bacterial components mediating mucosal inflammation of the respiratory tract. It appears that respiratory pathogens, including $H$. influenzae, stimulate the production of cytokines by respiratory epithelial cells in a way comparable with the stimulation in the urinary tract. Corticosteroids inhibit this proinflammatory response. It is suggested that the inflammatory process is similar to the stimulatory effect of cytokines released from inflammatory cells during infection. Bacterial products, including endotoxin, were shown to stimulate the response, although the discussion about the cell types involved in vivo continues.

At present, it is fair to conclude that bacterial pathogens of the respiratory tract have characteristics enabling them to colonize the mucosa of patients predisposed to these infections by underlying diseases, resulting in impaired host defence. During interaction of the bacteria with the mucous layer, and especially the epithelial cells, these bacteria stimulate an inflammatory response which is not effective in the eradication of the bacteria. The bacteria 
parasitize the host in a mild way, so that the host is not killed and the bacteria use the inflammatory conditions for their own benefit. There are indications that downmodulation of the inflammatory process may suppress bacterial infections, although we do not yet know how to achieve this, in such a specific way that the healing effect of the inflammatory response is not affected. The rapidly increasing knowledge of the inflammatory process and its mediators can be expected to provide new tools for effective anti-inflammatory treatment of infected patients. The prevention of bacterial adherence to the airway mucosa represents a challenge since it is desirable in order to avoid bacterial colonization of the respiratory tract.

\section{References}

1. Wilson R, Dowling RB, Jackson AD. The biology of bacterial colonisation and invasion of the respiratory mucosa. Eur Respir J 1996; 9: 1523-1530.

2. de Bentzmann S, Roger P, Puchelle E. Pseudomonas aeruginosa adherence to remodelling respiratory epithelium. Eur Respir J 1996; 9: in press.

3. Agace WW. The role of the epithelial cell in escherichia coli induced neutrophil migration into the urinary tract. Eur Respir J 1996; 9: in press.

4. Khair OA, Davies RJ, Devalia JL. Bacterial induced release of inflammatory mediators by bronchial epithelial cells. Eur Respir J 1996; 9: in press. 